\title{
Greening des Innovationssystems?
}

\author{
Das Thema Umwelt und Innovation läßt sich von zwei Seiten angehen. Die \\ innovationsökonomische Literatur legt als Ausgangspunkt das nationale Inno- \\ vationssystem nahe, dem eine Schlüsselrolle für die Leistungsfähigkeit hoch- \\ entwickelter Volkswirtschaften beigemessen wird. Die Umweltökonomie nähert \\ sich den Umweltinnovationen häufig von der Instrumentenseite. Beide Stränge \\ werden meist jedoch nicht miteinander verknüpft. Ein Forschungsprojekt an der \\ Fachhochschule für Wirtschaft versucht, diese Brücke zu schlagen (1).
}

$D$ Von Cbristian Rickert und Jan Nill er traditionelle Fokus des deutschen Innovationssystems liegt auf einer Verbesserung der Wettbewerbsfähigkeit durch Steigerung der Arbeitsproduktivität. Eine nachhaltige Entwicklung erfordert hingegen ein „Greening“ der Effizienzlogik des Innovationssystems im Sinne einer stärkeren Orientierung auf Ressourcenproduktivitätsfortschritte.

Von dieser Arbeitshypothese ausgehend wird untersucht, ob und inwieweit ein solcher Pfadwechsel des Innovationssystems seit dem 1. Ölschock 1973 bereits stattgefunden hat bzw. welchen Bedingungen er unterliegt. Weiter werden die Auswirkungen dieses ansatzweisen Pfadwechsels auf die Wettbewerbsfähigkeit einzelner Sektoren des verarbeitenden Gewerbes analysiert. Auf der Akteursebene sind Unternehmen, staatliche Forschungs- und Umweltpolitik und halbstaatliche Forschungseinrichtungen die zentralen Akteure für ein Greening des Innovationssystems. Erste Ergebnisse sollen hier skizzenhaft angedeutet werden.

\section{Die Rolle der Akteure}

Unternehmen tätigten 199762 Prozent der gesamten FuE-Ausgaben in Deutschland und führten zwei Drittel der Forschung selbst durch (2). Da Ausgaben für Umweltinnovationen nicht gesondert aufgeführt werden, dienen als Indikator für eine ökologischere Ausrichtung der Wirtschaft Unternehmensumfragen des ifo Instituts und des ZEW. In den alljährlichen Umfragen des ifo war in den 80er Jahren eine zunehmende Bedeutung des Innovationsziels „Reduzierung von Umweltbelastungen“ zu beobachten, während sie in den 90ern stagniert. Die seit 1993 durchgeführten ZEW-Untersuchungen zeigen hingegen, daß der Reduzierung der Umweltbelastung als strategisches Innovations- ziel in sämtlichen Branchen, mit Ausnahme der Chemischen Industrie, nur nachgeordnete Bedeutung beikommt. Das konkrete Unternehmensziel der „Entwicklung umweltfreundlicher Produkte" gehört jedoch in allen untersuchten Branchen zu den fünf wichtigsten (3).

Dieser Unterschied in der relativen Bedeutung zwischen der Verbesserung der Umweltbedingungen und dem angestrebten Produkterfolg kennzeichnet auch einen Wechsel der Ausrichtung der staatlichen Umweltpolitik. Systematische Umweltpolitik begann mit dem Umweltprogramm der Bundesregierung von 1971. Lange Zeit stand dabei das Ziel der Reduzierung von Umweltbelastungen durch die Entwicklung von „end-of-pipe“-Technologien im Vordergrund, diese machen zumindest bis Anfang der 90er Jahre den Löwenanteil der Umweltschutzinvestitionen der Industrie aus.

Im Zeitverlauf wurde zumindest programmatisch die Umwelt aus der "Opferrolle“ herausgelöst, durch kostensenkende und qualitätssteigernde Prozeß- und Produktinnovationen sollten makround mikroökonomische Interessen verbunden werden. Ins Zentrum rückte der integrierte Umweltschutz, wobei in den Umweltforschungsprogrammen, die seit 1984 in regelmäßigen Abständen aufgelegt wurden, lange Zeit der Schwerpunkt auf den Produktionsprozessen lag. Bis 1992 wurde z.B. praktisch überhaupt keine ökologische Produktentwicklung gefördert (4). Erst das Förderprogramm Nachhaltiges Wirtschaften von 1997 setzt hier deutlich veränderte Akzente.

Quantitativ stieg der Anteil der Umwelt- und Klimaforschung an den gesamten FuE- und Wissenschaftsausgaben des Bundes zwischen 1981 und 1997 von 4,07 Prozent auf 6,64 Prozent an. Schon die Relationen machen eine Grenze des Greenings deutlich; weiter haben Interviews mit relevanten Akteuren gezeigt, daß die Forschungspolitik hierbei eher eine passive Rolle gespielt hat und weitgehend den Schwerpunkten der Umweltpolitik gefolgt ist.

Im deutschen Innovationssystem spielen als dritte große Gruppe halb- bzw. nichtstaatliche Forschungseinrichtungen eine wichtige Rolle. Hier zeigt sich, daß Umweltschutz inzwischen als Thema durchaus aufgegriffen wird. Die auf angewandte Forschung ausgerichtete Fraunhofer-Gesellschaft wandte z. B. 1996 ca. 10 Prozent ihrer Ausgaben für Energie- und Umweltforschung auf. Eine eigenständige, aktive Rolle läßt sich jedoch bei den großen Forschungseinrichtungen nur begrenzt erkennen, die Vorreiterrolle bleibt hier kleineren Instituten vorbehalten.

Perspektivisch ergeben sich aus den bisherigen Ergebnissen zwei über das Projekt hinausreichende Ansatzpunkte, um die Möglichkeiten eines Pfadwechsels näher auszuloten:

- Erstens wäre es lohnenswert, das Zusammenspiel der Akteure und das Greening des Innovationssystems auch detailliert auf sektoraler Ebene zu analysieren, da zwischen den Sektoren durchaus markante Differenzen bestehen.

- Zweitens würde sich ein Vergleich der Entwicklung in verschiedenen Ländern auf europäischer Ebene anbieten, zumal Entwicklungen auf supranationaler Ebene die nationalen Innovationssysteme zunehmend ergänzen.

\section{Anmerkungen}

(1) Dos noch bis Ende dieses Jahres loufende Forschungsprojekt „Ökonomische Globalisierung, internationale Wettbewerbsähigkeit und notionale Innovationssysteme. Ökologische Innovationspolitik als Standorffaktor?" unter Leitung von Prof. Kurt Hübner wird im Auftrag des Bundesforschungsministeriums durchgeführt.

(2) Aktuelle Daten finden sich im vom BMBF herausgegebenen Foktenbericht 1998 zum Bundesbericht Forschung. (3) Vgl. z.B. Adler, U.: Integrierter Umwellschutz als Beispiel zukunffstähiger Innovation. Ifo-Schnelldienst 17 . $18 / 1997$, S. 46 und

Felder, J. et al.: Innovationsverhalten der deutschen Wirtschaft, ZEW, Mannheim 1994, S.28.

(4) Vgl. Angerer, $G$. et al.: Umwelttechnologie am Standort Deutschland, Heidelberg 1997, S.43.

\section{Die Autoren}

Christian Rickert und Jan Nill sind wiss. Mitarbeiter an der Fachhochschule für Wirtschaft Berlin. Kontakt: Badensche Str. 50-51, 10825 Berlin, Tel. 030/ 85789125, E-mail: ricker!@fhw-berlin.de, jinill@fhw-berlin.de bzw. Jan.Nill@ioew.de 
(c) 20I0 Authors; licensee IÖW and oekom verlag. This is an article distributed under the terms of the Creative Commons Attribution Non-Commercial No Derivates License (http://creativecommons.org/licenses/by-nc-nd/3.o/), which permits unrestricted use, distribution, and reproduction in any medium, provided the original work is properly cited. 